\title{
Evaluation of a Community Dental Service for Homeless and 'Hard to Reach' People
}

Sue Caton (PhD, Research Associate, Research Institute for Health and Social Change, Manchester Metropolitan University)

Faye Greenhalgh (Project Manager, Revive Dental Care)

Lynne Goodacre (PhD, Assistant Director, NHS R\&D NW) 


\section{Abstract}

Objective: Since 2013, Revive Dental Care has been operating a community outreach dental service for homeless and 'hard-to-reach' patients. This research aimed to a) explore the dental care experienced by people accessing the service $b$ ) examine barriers and facilitators to using a dental service c) examine the impact of the service d) identify good practice in providing dental services for homeless people.

Methods: Semi-structured interviews with 20 patients, 9 members of the dental staff and 4 staff members from the community centres providing services for homeless people.

Results: Findings suggest that homeless patients have overall poor daily dental care and experience significant dental problems due to a range of lifestyle factors. Most participants had not seen a dentist for many years and previous experiences of seeing a dentist were often unpleasant. Barriers to care included fear, embarrassment, lack of money, living chaotic lifestyles, not prioritising dental care and difficulties finding an NHS dentist that would take on homeless people. Service provision for homeless and/or hard-to-reach patients needs to be proactive with dental staff going to community settings and making personal contact.

Conclusion: Crucially, providers must acknowledge that the patients are vulnerable. A successful service needs to be informal, adapt to patient needs and accommodates chaotic lives. 


\section{Introduction:}

In 2004, the British Dental Association (BDA) published a report setting out the case for improving dental health services for people who are homeless ${ }^{1}$ which highlighted the potentially negative impact that attitudes towards dental health may have on people accessing dental care.

The population of people who are homeless is difficult to define due to the range of definitions used ${ }^{2}$ with people sleeping rough, representing only a small proportion of the population. In 2013/14, 111,960 people in England contacted Local Authorities for assistance ${ }^{3}$ but in addition, 'hidden homelessness' includes people either sleeping in hostels or living with friends.

Regardless of how this population is defined there is significant evidence regarding the level of need for dental treatment $(2,456)$. Factors contributing to poor dental health include poor nutrition and living conditions, substance and alcohol misuse, smoking and poor mental health.

Two studies have undertaken retrospective case note and record card reviews $(4,6)$ and provided insights into targeted services for homeless people. Both studies highlight some of the challenges of providing services to this population including high levels of non-attendance and low levels of completion.

The need to deliver flexible services addressing the needs of homeless people has been highlighted consistently including developing outreach services with the ability to refer into mainstream practice when treatment is required. Whilst some research has previously been undertaken to evaluate dental services for homeless people, one of the elements missing is that of the patients' voice. 
Since 2013, Revive Dental Care has been operating a community outreach dental service for 'hard-to-reach' clients living in Greater Manchester.

Service users are predominantly homeless or from disadvantaged

backgrounds and have complex health needs which include substance and alcohol misuse.

Based initially in a health centre incorporating a GP's surgery contracted to provide health services for homeless patients, the service evolved to working with community drop-in centres used by this population. A general dental practitioner (GDP) and/or a dental nurse now visit drop-in centres on a regular basis and conduct a general examination. If further treatment is required, clients are referred to a weekly clinic in the practice.

\section{Methodology}

The research was located within a qualitative phenomenological design to develop a greater understanding of the experiences of both service users and providers. The aims of the study were to:

1. Explore the dental care experienced by people accessing the service

2. Examine barriers and facilitators to using a dental service

3. Examine the impact of an outreach dental service

4. Identify good practice in setting up and providing outreach dental services

Ethical approval was granted by Manchester Metropolitan University and informed consent was taken from each participant. Participants were informed that the name of the dental practice may be used in dissemination but data from interviews would be anonymised.

Semi-structured interviews (using an interview schedule) were carried out with 33 participants. Participants fell into one of three categories: 
1. Patients attending the clinic. A convenience sampling method was used over four months, with patients attending clinic being given information and asked if they would like to participate. Seventeen male patients and three females took part in short semi structured interviews that took place immediately before or after their appointment at the clinic. Sampling continued until data saturation was reached at twenty participants.

2. Nine members of staff who were most closely involved in providing the service including management, GDPs, and dental nurses took part in semi-structured interviews.

3. Four staff members from the community centres providing services for homeless people took place in semi-structured interviews which took place at the community centres (one was a telephone interview).

\section{Analysis}

Descriptive data was collated from practice records. Interviews were transcribed verbatim and analysed thematically (Braun \& Clarke, 2006). Coding of the interview transcripts was carried out by both authors.

\section{Results}

Data from practice records, as shown in Table One, demonstrate that over a six month period, the dental team made 20 site (community centre) visits (averaging 3.33 per month) and saw 103 patients. Failure to attend clinic was high with 106 patients not attending over this period.

\section{Insert Table One about here}

Table Two gives details of the routes into the service described by the participants.

\section{Insert Table Two about here}


Through thematic analysis of transcripts, we identified five global themes: Dental Experiences; Reaching Out; Accommodating Chaotic Lives; Behaviour Change; Looking Forward.

\section{Dental Experiences}

'Dental Experiences' examines how the participants experienced dental care prior to engagement with the service exploring impact of lifestyle on dental care and going to the dentist.

Dental care undertaken by participants prior to attending the clinic was varied, with the majority of participants speaking of poor daily dental care:

I've spent a lot of time living on the streets over the years and to be honest with you oral hygiene is not high up on the agenda to be honest with you. You know getting from day to day is more important. Patient 18

The majority of the patients that attended the clinic were, or had been involved in alcohol or substance misuse, which impacted upon their oral health. Poor diet and fighting were also mentioned as having caused damage:

... the majority of them, I'd probably say ninety percent of the patients, people that come in, clients, have stubs for teeth and that's just heroin, cocaine, has totally destroyed teeth and now they have just got stubs and essentially they need them taking out and dentures. Dental Staff

Most patients explained that prior to attending clinic, they had not visited the dentist for many years. Two participants had attended more recently when in prison. Twelve of the 20 patients spoke about the negative influence of previous bad experiences, two had attempted to attend a dentist but had missed appointments causing de-registration. Other barriers discussed were embarrassment about the condition of their mouths and difficulty finding an NHS dentist that would take on homeless people.

Prior to the service being available, participants relied on ad hoc solutions. Staff from the community centres described relying on sympathetic dentists, or more commonly, the emergency dental service. Some of the patients said that without the 'Hard to Reach' service they would have "left it".

With regard to dealing with any dental pain prior to accessing the service, a number of approaches were described: 
I'd have took heroin and I'd have took painkillers and if I'd come to the point of - I'd wait till it was an emergency. That's what I did with everything, I waited till it was an emergency and then I dealt with it if that happened. Patient 7

Drank more, it takes away the pain. Eat Paracetamol, it takes away the pain. Generally ignore it. I've had abscesses over the years and I just pop them myself and carry on, it doesn't bother me. ............... it's easier than trying to go through the system... Patient 18

The number of times l've been asked for a pair of pliers because someone wants to pull their own teeth, you know, l've lost count which is awful. Community Centre Staff

\section{Reaching Out}

Reaching out to people and to the community was identified as the key to the success of the service. Currently ten community centres are visited on a regular basis. According to size and need, sites are visited between twice a year and up to once a month. The success of the service was highlighted consistently as being down to the initial contact being made in a non-threatening environment that the patients are familiar with.

I think the fact that they've seen them here, because people trust here, they trust staff here and they feel comfortable here and they access it quite regularly then if somebody is here by association there is already a level of trust in place. Community Centre Staff

The location of the clinic alongside a GP providing services for homeless patients was also significant and appeared to assist many patients in engaging with the service. Six patients mentioned that the GP had referred them, or that they were aware of the dentist because they attended the GPs clinic.

Five participants came to the clinic with a friend and from all of the patients interviewed only one had not been told by someone else about the service emphasising the importance of word of mouth within this community.

\section{Accommodating Chaotic Lives}

Accommodating Chaotic Lives brings together a number of attributes of the service which demonstrate its responsiveness to the needs of this community. 
Flexible Appointments: The community centre visits were primarily for signposting to the clinic. Patients were then given an appointment at the clinic. The chaotic nature of people's lives, as well as factors such as the weather influenced clinic attendance along with the presence or absence of pain:

It's about 'today', you can't wait for next week because if you haven't got the pain today and your appointment's today they won't go, don't ask me why but that's just the way they live. But next week they'll have the pain again and they might come in and say "Ouch." And I'll say "Did you go to the dentist?" "No, I missed it". "Why did you miss it?" "I was alright then". Community Centre Staff

The service has therefore adapted to accommodate haphazard attendance.

we accommodate for chaotic lives, if they fail they fail. I would like them not to, and I do warn them saying - "Please come to your appointment" - or if they fail "Don't fail again". But if they are in pain or they want to rebook their appointment they are more than welcome to. Dental Staff

To assist the smooth running of the service, members of the dental team telephone on the morning of the appointment as a reminder. Many patients referred to having missed a previous appointment, but the fact that they returned demonstrates the need for this flexibility.

Yes I am having a course of treatment, I don't go anywhere else to be honest with you because I have got a habit of missing my appointment you know, I forget, and I think they are quite lenient here so that's why I keep coming back here. And the treatment's really good as well. Patient 3

Whilst not encouraged, patients can also drop into the clinic which addresses the immediacy of peoples' need.

\section{Overcoming Language Barriers and Anti-Social Behaviour The}

dental team had to adopt strategies to deal with various characteristics that were common amongst this patient population. For example, increasing numbers of non-English speakers presenting for treatment led to the use of a telephone interpreting service, Language Line. 
The staff had also developed strategies to respond to patients under the influence of alcohol or drugs (which raised concerns around consent and medical safety associated with carrying out invasive treatments), displaying aggressive behaviour, or wanting to sleep or stay for extended periods in the waiting room. Whilst the team almost always saw a patient under the influence of drugs or alcohol no treatment would be given.

Approachable, Friendly Staff - The dental staff were described consistently as: good communicators, approachable, proactive, supportive, friendly, open-minded, compassionate, not easily shocked and sympathetic.

They're so understanding, no pressure, you just feel at ease when you are here...............I'm coming back here because they're understanding and gentle with you, and they explain everything for you and you get a right to say how you feel and how you want to be treated, and they do it. Patient 20

The dental staff and community centre staff agreed that being approachable and informal were key characteristics to the success of the service.

I think to run a service like this you need someone ....... who will get out there in a room and talk to people and be approachable and you know really motivate people to doing something about their dental health care. I mean we can make people appointments and try to encourage people to come along and they do so, but on the day of the surgeries ... (person's name) out there around the room, you know, getting more people through the door who perhaps don't have the confidence to come and ask. Community Centre Staff

Working with the Benefits System - Most people accessing the service were claiming state benefits and therefore eligible for free dental treatment. However, some patients were not in receipt of benefits but could not afford to pay for treatment. The dental team estimated that this 
situation arose around once a month describing how if the person was in pain, the practice had taken the decision to cover the cost.

\section{Behaviour Change: "I'm going to reform"}

Behaviour Change describes the impact of the service in two ways. Firstly, with regard to patients lifestyle changes. Whilst many patients were referred initially because of pain, missing or broken teeth, taking the first step of going to the dentist led to an aspiration of 'sorting their teeth out'. Patients also talked about more specific aspects of change that they aspired to such as: wanting to eat without discomfort, wanting the selfconfidence they knew repaired teeth would bring, wanting to seek employment and wanting to develop personal relationships. Some of these reasons were linked to a number of patients being in the process of 'turning their life around'.

My self-confidence really, it will do wonders for that. There's nothing worse than talking to people in the street and talking to them keeping your mouth covered so they can't see the damage that has been done. And as I say it will help greatly with my confidence with the chances of getting work. Patient 19

Basically my teeth are in a shocking state and I need to get false teeth put in because I find it hard to approach women with the state of my face as it is, because a lot of women wouldn't like talking to me, because of my terrible gungy teeth I've got. Patient 11

Secondly, 'Behaviour Change' was related to dental attendance. An ultimate criteria of success for the service was that it led to longer-term engagement with dental care.

I actually really strongly believe that it's about behavioural change. Yes you've got patients that you might be able to catch but I don't want to just catch them I want it to be a life-changing thing that we actually make a big difference to the patients. Dental Staff

For many patients, making the first appointment created a resolve to continue treatment. Some were part way through treatment and committed to completing it, most expressed a commitment to more 
regular check-ups, or at the very least, returning if they needed treatment for pain. Some made comments implying an increase in daily dental care:

It was more like once a day before I was coming here but they were like - "you need to brush your teeth two times a day because we need to get these sorted out" and what not. So I've been doing it, just following the plan. Patient 16

However, there was some evidence to suggest that the reality of these changes may not be as positive and long standing as some of patients implied. Many partially made sets of dentures had never been completed and it was seen as unusual for patients to return for regular check-ups.

\section{Looking Forward}

Looking Forward explores views on ways in which the service could be developed. Opinion was divided about whether it would improve the service to provide dental treatment at the community drop in centres. Advantages of this model were described as replacing the need for patients to attend clinic at a later date. However, members of the dental staff highlighted challenges around access to equipment, patient safety, and cross infection.

There was discussion relating to developing a nurse led, rather than dentist led service given that no treatment is given at the community sites. Increasingly this was how the service was developing with the project manager (a dental nurse) visiting sites unaccompanied. Community centre staff, however, valued seeing the dentists.

Scope for the clinics to be busier was highlighted and this was observed during the fieldwork (although it must be acknowledged that it is quite complex due to unpredictable numbers attending, see Table One). Suggested approaches to achieving this included: increasing the number of visits to the community centres, increasing the number of clinics, a longer clinic day, or reverting to holding the clinic at the same time as the GPs drop in session, increasing publicity.

...if it was more well known to people that it was available, because I've been homeless for years on and off and I didn't know anything like this existed. So if there was more publicity, if it was pushed out there a bit more a lot more people would actually come to it. Patient 18

The service was described by a member of the dental team as being "the one area of our business that probably is least influenced by income". The dental team view the value of the service as being about developing the 
practice, incorporating dentistry that is stimulating, interesting and rewarding. The team universally enjoyed their involvement in the service:

Changing someone's life. When someone comes in with stumps, upset, can't smile, doesn't feel confident speaking to someone and then they walk out with new teeth. Or someone that has changed in another aspect of their life, i.e. given up drinking, when they've been an alcoholic but because they've had so many dental treatments you've seen that turnaround and I find that rewarding.

Dental Staff Discussion \& Conclusion

Our findings reflect previous studies $\left(2,{ }^{7}\right)$ by showing that patients' lifestyle in terms of diet and alcohol and substance misuse have a significant negative impact on their oral health. We have also found, in line with previous research, that failure to attend dental services is high. Whilst this was due in part to the services inability to accommodate the chaotic lives of this patient population, our research highlighted the complex needs of this group, combined, often with apparent low selfesteem. A significant barrier to attending a dental service was that when people are struggling with homelessness and perhaps drug or substance addiction, dental care is simply not high on their priorities until they experience pain, at which point it becomes urgent. Many of our participants spoke of previous bad experiences at the dentist and again this is in line with previous research which suggests that dental anxiety amongst this population is higher than average (2).

Our data suggest that without services specific to the needs of homeless people, pain associated with dental problems can mean that people take their own courses of action (which included resorting to alcohol and substance use). For some people, our findings suggest that the service can have a significant impact by removing pain and increasing selfconfidence.

The findings demonstrate the importance of a service that exploits personal contacts. Most patients came to the clinic following a conversation with a member of the dental team at a community centre, others came with friends who had been before, others came on the recommendation of staff from the community centres.

Overall, the study highlighted the crucial importance of a patient needs led service where the providers have recognised the importance of going into community settings, talking to people and getting people into the system. Vitally, the target population for this service are vulnerable people, often lacking in confidence and the best way to access them is to 
actually go out and get them. The service has developed around the principle of accommodating chaotic lives; adapting to the needs of the patients.

\section{Acknowledgements}

We would like to acknowledge the staff at Revive Dental Care, the patients from the 'hard-to-reach' service and staff from the community centres. This project was funded by Health Education North West. 
References 
1 British Dental Association. Dental care for homeless people. Policy statement. London: British Dental Association, 2004.

2 Collins J., Freeman R. Homeless in North and West Belfast: an oral health needs assessment. British Dental Journal. 2007: 202:12:E31

3 Crisis/ Definition and number of homeless people: http://www.crisis.org.uk/pages/homeless-def-numbers.html accessed 17/6/2014

4 Daly, B. J., Newton, J.T., Batchelor, P. Patterns of dental service use among homeless people using a targeted service. Journal of Public Health Dentistry. 2010; 70:1: 45-51

5 Hill, K. B., Rimington, D. Investigation of the oral health needs for homeless people in specialist units in London, Cardiff, Glasgow and Birmingham. Primary Health Care Research \& Development. 2011; 12: 135-144

6 Simons, D., Pearson, N., Movasaghi, Z. Developing dental services for homeless people in East London. British Dental Journal. 2012: 213:7:E11 doi: 10.1038/sj.bdj.2012.891

7 Coles, E., Watt, C., Freeman, R. (2012) 'Something to Smile About': An evaluation of a capacity-building oral health intervention for staff working with homeless people. Health Education Journal, 72, 2, 146-155 
Table One: Numbers of Patients Seen by 'Hard-to-Reach' Service

\author{
2014 \\ No of Site Visits \\ Patients Seen \\ at community sites \\ Patients seen at clinic \\ Failure to Attend
}

\title{
April-September
}

103

213

106

Table Two: Referral Routes into the Service

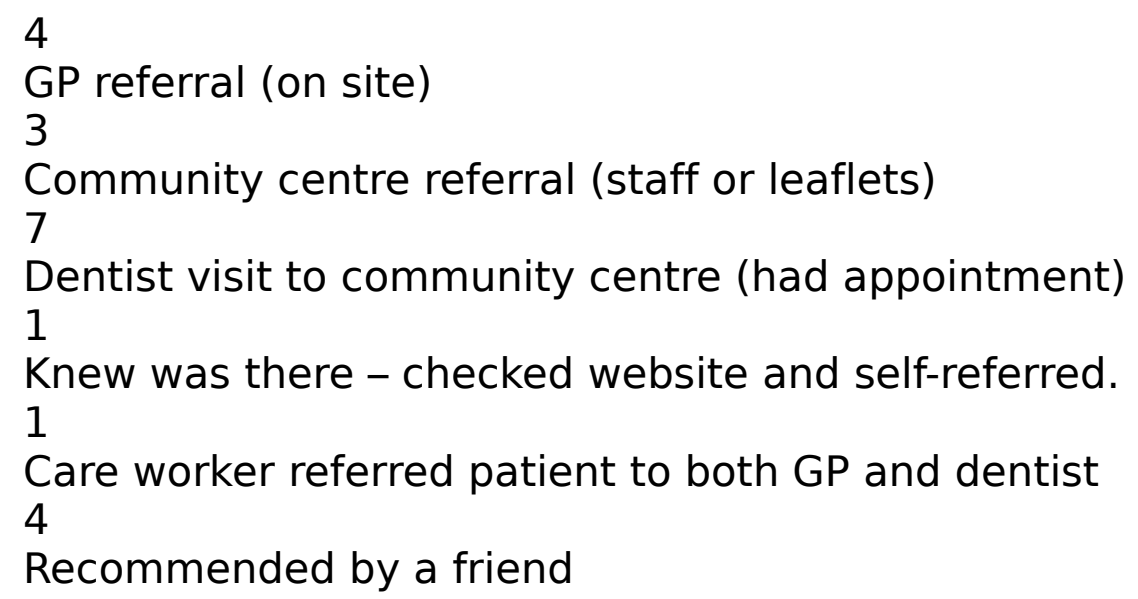

\section{Referral Route}

